In contrast to the version of this article initially published, the authors find that there is now no evidence to support the association with esophageal squamous cell carcinoma susceptibility of rs17761864[A] at 17p13, rs6503659[A] at 17q21 or rs2847281[C] at 18p11 in the original data, in two independent sets of cases and controls collected in other Chinese populations or in the joint analysis of three studies.

The associations originally published appear to be due to inadequate control for population stratification. A reanalysis by the authors of these data resulted in corrected estimates for these SNPs in Beijing genome-wide association data (Nat. Genet. 46, 1001-1006, 2014). For more information on the role of the correction for population stratification in the differences between the previously published estimates for the three SNPs rs6503659, rs17761864 and rs2847281, see Supplementary Figure 8 and Supplementary Table 8 in the new publication (Nat. Genet. 46, 1001-1006, 2014).

The error has been corrected in the HTML and PDF versions of the article. In addition, Supplementary Figure 1 and Supplementary Tables 1 , 3, 5 and 7 have been corrected.

\title{
Corrigendum: Genome-wide association study of esophageal squamous cell carcinoma in Chinese subjects identifies susceptibility loci at PLCE1 and C20orf54
}

Li-Dong Wang, Fu-You Zhou, Xue-Min Li, Liang-Dan Sun, Xin Song, Yan Jin, Jiang-Man Li, Guo-Qiang Kong, Hong Qi, Juan Cui, Lian-Qun Zhang, Jie-Zhi Yang, Ji-Lin Li, Xing-Chuan Li, Jing-Li Ren, Zhi-Cai Liu, Wen-Jun Gao, Ling Yuan, Wu Wei, Yan-Rui Zhang, Wei-Peng Wang, Ilyar Sheyhidin, Feng Li, Bao-Ping Chen, Shu-Wei Ren, Bin Liu, Dan Li, Jian-Wei Ku, Zong-Min Fan, Sheng-Li Zhou, Zhi-Gang Guo, Xue-Ke Zhao, Na Liu, Yong-Hong Ai, Fang-Fang Shen, Wen-Yan Cui, Shuang Song, Tao Guo, Jing Huang, Chao Yuan, Jia Huang, Yue Wu, Wen-Bin Yue, Chang-Wei Feng, Hong-Lei Li, Yan Wang, Jin-Ya Tian, Yue Lu, Yi Yuan, Wen-Liang Zhu, Min Liu, Wen-Jing Fu, Xia Yang, Han-Jing Wang, Suo-Li Han, Jie Chen, Min Han, Hai-Yan Wang, Peng Zhang, Xiu-Min Li, Jin-Cheng Dong, Guo-Lan Xing, Ran Wang, Ming Guo, Zhi-Wei Chang, Hai-Lin Liu, Li Guo, Zhi-Qing Yuan, Hai Liu, Qin Lu, Liu-Qin Yang, Fu-Guo Zhu, Xiu-Feng Yang, Xiao-Shan Feng, Zhou Wang, Yin Li, She-Gan Gao, Qirenwang Qige, Long-Tang Bai, Wen-Jun Yang, Guang-Yan Lei, Zhong-Ying Shen, Long-Qi Chen, En-Min Li, Li-Yan Xu, Zhi-Yong Wu, Wei-Ke Cao, Jian-Po Wang, Zhi-Qin Bao, Ji-Li Chen, Guang-Cheng Ding, Xiang Zhuang, Ying-Fa Zhou, Hou-Feng Zheng, Zheng Zhang, Xian-Bo Zuo, Zi-Ming Dong, Dong-Mei Fan, Xin He, Jin Wang, Qi Zhou, Qin-Xian Zhang, Xin-Ying Jiao, Shi-Yong Lian, Ai-Fang Ji, Xiao-Mei Lu, Jin-Sheng Wang, Fu-Bao Chang, Chang-Dong Lu, Zhi-Guo Chen, Jian-Jun Miao, Zeng-Lin Fan, Ruo-Bai Lin, Tai-Jiang Liu, Jin-Chang Wei, Qing-Peng Kong, Yu Lan, Yu-Jing Fan, Fu-Sheng Gao, Tian-Yun Wang, Dong Xie, Shu-Qing Chen, Wan-Cai Yang, Jun-Yan Hong, Liang Wang, Song-Liang Qiu, Zhi-Ming Cai \& Xue-Jun Zhang

Nat. Genet. 42, 759-763 (2010); published online 22 August 2010; corrected after print 27 August 2014

In contrast to the version of this article initially published, the authors now find no evidence to support association with esophageal squamous cell carcinoma susceptibility for rs13042395[T] at 20p13 in their original data, in two independent sets of cases and controls collected in other Chinese populations or in the joint analysis of these three studies.

The original signal at the C20orf54 locus (rs13042395) in the published Henan genome-wide association study (GWAS) was based on the scan of a total of 2,810 subjects $(1,077$ cases and 1,733 controls) with a per-allele odds ratio (OR) of $0.66(95 \%$ confidence interval $(\mathrm{CI})=0.58-0.74$; $\left.P=1.51 \times 10^{-11}\right)$, and $P<5 \times 10^{-6}$ was reported for a genetically matched subset of 937 cases and 692 controls.

A new analysis by the authors (see Supplementary Table 8 in Nat. Genet. 46, 1001-1006, 2014) included a distinct genetically matched subset of the original genotypes ( 1,789 total subjects: 1,076 cases and 713 controls; $\mathrm{OR}=0.80(95 \% \mathrm{CI}=0.65-0.98) ; P=0.03)$ and subjects scanned after publication of the initial Henan data (669 new subjects: 229 cases and 370 controls; $\mathrm{OR}=1.06(95 \% \mathrm{CI}=0.82-1.38)$; $P=0.63)$. The combination of the genetically matched and new subjects $(n=2,458$ subjects in total: 1,375 cases and 1,083 controls $)$ yielded OR $=0.88(95 \%$ CI $=$ $0.76-1.02 ; P=9.12 \times 10^{-2}$ ).

Thus, the original Henan scan is the only result that showed a significant association for rs 13042395 . None of the three new analyses performed confirmed a significant association for rs13042395. Moreover, this SNP was not significant in the National Cancer Institute GWAS (OR $=0.95$, $\left.95 \% \mathrm{CI}=0.86-1.05 ; P=3.04 \times 10^{-1}\right)$, Beijing GWAS $\left(\mathrm{OR}=1.07,95 \% \mathrm{CI}=0.93-1.23 ; P=3.34 \times 10^{-1}\right)$ or combined data $(\mathrm{OR}=0.96,95 \% \mathrm{CI}=$ $0.90-1.03 ; P=3.02 \times 10^{-1}$; see Supplementary Table 8 in Nat. Genet. 46, 1001-1006, 2014).

In summary, the published association for rs13042395 did not replicate in additional analyses of data from Henan. Nor did it replicate in a GWAS from a similar high-risk population (National Cancer Institute) or in a GWAS from a low-risk population (Beijing). Thus, the original finding was likely the result of inadequate control for population stratification using the genetically unmatched subjects or, less likely, could have been due to chance alone.

The error has been corrected in the HTML and PDF versions of the article. In addition, Supplementary Figures 1-4 and Supplementary Table 1 have been corrected. 